\title{
Umbral Anaeróbico. Problemas conceptuales y aplicaciones prácticas en deportes de resistencia Anaerobic Threshold. Conceptual problems and practical applications in endurance sports Emilio Fernandez Rodriguez, Óscar Romero Ramos, Rafael Merino Marbán, Alejandro Cañas del Palacio Universidad de Málaga (España)
}

Resumen. El objeto de este artículo fue mostrar el excesivo entramado terminológico relacionado con el entrenamiento del umbral anaeróbico (UAN). Faude, Kindermann y Meyer (2009) encontraron 25 acepciones diferentes sobre el termino umbral. A pesar de ello, se dan unas pautas didácticas sobre diversos aspectos relacionados con su entrenamiento (pruebas de esfuerzo, frecuencia cardiaca, factores de rendimiento, planificación...). Se describe como entrenan el UAN los mejores fondistas del mundo, como lo distribuyen en zonas de entreno o, en qué parte de la temporada lo aplican en función de los modelos de planificación más habituales. En general, los fondistas de máximo nivel lo aplican de forma polarizada y en poca cantidad respecto al volumen total del año y a otras zonas de entrenamiento.

Palabras clave: umbrales, entrenamiento de resistencia, planificación, lactato.

Abstract. The purpose of this paper was to highlight the excessive terminological framework related with anaerobic threshold (ANt). Faude, Kindermann and Meyer (2009) found 25 different definitions about the term threshold. Despite this, it is possible to give didactic guidelines on various aspects related to their training (lab test, heart rate, factors of performance, scheduling). These guidelines show how the best athletes in the world train the ANt, how they distribute it in training zones, or in what part of the season they apply it according to the most usual planning models. In general, the top-level runners apply it in a polarized way, as well as in a small amount with respect to the total volume of the year and other training zones. Key words: thresholds, endurance training, schedule, zones, lactate.

\section{Introducción}

Wasserman y Mcllroy (1964), primeros en definir el concepto de $\mathrm{UAN}$, indicaban que era la tasa de trabajo del consumo de oxígeno $\left(\mathrm{VO}_{2}\right.$ a partir de la cual se produce una acidosis metabólica. Este concepto fue diversificándose y dio lugar a una proliferación de términos ambiguos. Ello dependió de la formación, metodología o protocolos empleados. Actualmente existe un crisol de métodos, palabras y siglas. Hay desacuerdo en la comunidad científica sobre estos aspectos y se produce por la ausencia de estandarización metodológica y falta de consenso sobre la base teórica del concepto en sí.

Dentro de esa amalgama de términos y a efectos de una mayor aclaración posterior, Svedahl y Macintosh (2003) señalan los más comunes:

-Umbral anaeróbico o intensidad de ejercicio por encima de la cual, hay una contribución neta de energía asociada a la acumulación de lactato.

-Estado de equilibrio máximo de lactato (MLSS) o la mayor intensidad de ejercicio en la que, la concentración de lactato en sangre no aumenta más durante el ejercicio de carga constante.

-Umbral de lactato (UL) o intensidad del ejercicio que se asocia con el primer aumento sustancial en el lactato sanguíneo durante un ejercicio incremental. Se utilizan varios criterios específicos para identificar este aumento, y algunos tienen su propio nombre.

-Umbral anaeróbico individual (UANI). Versión especial de un umbral de lactato o la intensidad del ejercicio identificado por una línea trazada a partir de una concentración de lactato de recuperación, tangentea la concentración de lactato observada durante una prueba incremental.

-Inicio de la acumulación de lactato en la sangre u OBLA. Intensidad del ejercicio a la cual la concentración de lactato en sangre alcanza 4 mmol-L-1. durante una prueba de ejercicio incremental.

-Umbral de ventilación o intensidad del ejercicio en el que el aumento de la ventilación se vuelve desproporcionado al aumento en potencia o velocidad de locomoción durante una prueba de ejercicio incremental.

-Lactato mínimo o velocidad mínima de lactato. Es la velocidad en la que el lactato sanguíneo alcanza un valor mínimo durante un ejercicio incremental que se inicia en presencia de acidosis láctica.

Tradicionalmente ha habido dos grupos terminológicos de umbrales vinculados a lo aeróbico o anaeróbico. En uno se encuentran los que señalan el inicio del incremento de la producción de lactato:

Fecha recepción: 23-11-17. Fecha de aceptación: 15-11-18 Oscar Romero Ramos

oromero@uma.es
- El punto de óptima eficiencia respiratoria de Hollmann.

- El umbral de metabolismo anaeróbica de Wasserman y Mcllroy. - El umbral aeróbico de Kindermann.

- El OPLA o comienzo de acumulo de lactato en plasma de Farrell.

- El umbral láctico de Davis.

- La transición anaeróbica individual de Pesenhofer.

- El umbral ventilatorio 1(VT) de Orr.

El segundo, recoge conceptos vinculados al incremento de la intensidad de la transición de una carga de trabajo tolerable, a una más intensa que se pueda mantener. Londeree y Ames, en 1975 lo denominaron Maximum Lactate Steady State (MLSS). Otros términos similares son:

- El umbral aeróbico-anaeróbico de Mader.

- El umbral anaeróbico de Kindermann.

-El IAT oumbral anaeróbico individual de Keul en 1979y Stegmann. - El OBLA o inicio del acumulo de lactato en sangre de Sjödin y Jacobs.

- El umbral ventilatorio $2\left(\mathrm{VT}_{2}\right)$ de Orr.

El primer grupo esta en niveles del 65-80\% del máximo consumo de oxígeno $\left(\mathrm{VO}_{2 \text { máx }}\right)$ con una intensidad de ejercicio en $2 \mathrm{mmol} \cdot \mathrm{L}-1$ de concentración de lactato. El segundo grupo esta en el 80-95\% del $\mathrm{VO}_{2}$ máx (equivalente teórico a $4 \mathrm{mmol} \cdot \mathrm{L}-1$.). Se observa el uso indistinto de mas palabras aeróbico-anaeróbico en ambos grupos. El uso de estos dos conceptos no es correcto. Chamari y Padulo (2015) indicaban que sus significados presentan problemas referentes a la presencia o ausencia del $\mathrm{O}_{2}$ en las dos vías. Hogan (2001) estableció que el metabolismo de la presencia/ausencia de oxígeno (y del consumo de oxígeno) no es el que se pensaba. Los valores del umbral aeróbico (UA) y UAN se han venido estableciendo, como válidos, en 2 y $4 \mathrm{mmol} \cdot \mathrm{L}-1$. Stegmann y Kindermann (1982) señalaban que el UAN ocurría en diferentes niveles de lactato para diferentes atletas y, el uso de $4 \mathrm{mmol} \cdot \mathrm{L}-1$. para todos, podía ser engañoso ya que el umbral anaeróbico individual (UANI) suele tener un rango de dos a seis $\mathrm{mmol} \cdot \mathrm{L}-1$. Ese valor teórico no tiene en cuenta la variabilidad interindividual y puede no ser objetivo, es decir, puede no representar la misma intensidad de ejercicio en corredores de distinto nivel o modalidad atlética.

En el plano práctico deportivo, es recurrente utilizar ambos términos (aeróbico-anaeróbico) de forma confusa. En natación, para entrenar el UAN se sitúa a éste, en la zona de entrenamiento (ZETO) llamada aeróbico medio o zona dos. El UAN es una ZETO más suave (a pesar de incluir la palabra anaeróbico) que la del $\mathrm{VO}_{2 \operatorname{máx}}$ y este, sin embargo, está considerado como entrenamiento (ETO) aeróbico en su zona mayor, siendo denominado en algunos deportes como ETO aeróbico intenso o zona tres. Para mayor confusión Sergeyevich-Mishchenko y Dmitriyevich-Monogarov (2001) denominaron al UA como primer 
umbral anaeróbico $\left(\mathrm{UAN}_{1}\right)$ y al anaeróbico como segundo umbral anaeróbico $\left(\mathrm{UAN}_{2}\right)$ o umbral anaeróbico real (UANr).

Según Santos-Concejero et al. (2013) «se han propuesto diferentes índices de lactato en sangre (...), como el umbral de lactato (UL), el MLSS o, la aparición de acumulación de lactato en sangre (OBLA)» (p.67). Hall, Rajasekaran, Thomsen y Peterson (2016) dibujan de esta manera el problema terminológico-conceptual:

Los términos «umbral de lactato», «umbral anaeróbico», «umbral aeróbico», «punto de inflexión del lactato», «inicio de la acumulación de lactato en sangre (OBLA)» $\mathrm{y}$ «máximo estado de equilibrio de lactato (MLSS)» se usan indistintamente, aunque los significados son diferentes. Estos términos se refieren a dos fenómenos específicos. Primero, durante un ejercicio incremental, hay un punto en el que el lactato sanguíneo comienza a aumentar por encima de los valores de reposo. Este punto fue descrito como «umbral anaeróbico» por Wasserman, pero como «umbral aeróbico» por Kindermann. Actualmente, en la mayoría de la literatura científica, este punto se denomina «umbral de lactato aeróbico», [ $\left[\mathrm{UL}_{1}\right]$ pero para mayor claridad llamaremos a este punto «inicio de la acumulación de lactato» (OBLA). En segundo lugar, existe una intensidad de ejercicio casi máxima, por encima de la cual es inevitable un aumento continuo en el lactato sanguíneo. Kindermann llamó a este punto el «umbral anaeróbico» y la mayoría de la literatura científica ahora, se refiere a él como el «umbral del lactato anaeróbico» [UL $]$. Sin embargo, en aras de la claridad llamaremos a este punto el «máximo estado estable del lactato» (MLSS) (...). Algunos investigadores recomiendan usar niveles de lactato sanguíneo fijo (por ejemplo, la carga de trabajo que produce un lactato sanguíneo de $2 \mathrm{mmol} \cdot \mathrm{L}-1$. podría correlacionarse con OBLA, y la carga de trabajo que produce una de $4 \mathrm{mmol} \cdot \mathrm{L}-1$. podría correlacionarse con MLSS) para estimar OBLA y MLSS, pero la evidencia demuestra que estos métodos son malos indicadores de estos fenómenos fisiológicos. A pesar de ello, estos métodos se usan comúnmente en pruebas comerciales de resistencia. (p.12)

Algunos autores consideran más umbrales. Parten de los valores de lactato en reposo y hablan de porcentajes con respecto al mismo. Así en el UA o UAN, , el valor de ácido láctico sería el doble del valor de reposo del deportista evaluado. Incrementar la carga de trabajo produciría un aumento del ácido láctico, punto en el que, algunos investigadores sostienen que el valor en sangre de lactato sería aproximadamente de 4 mmol·L-1. o, UAN o, segundo umbral anaeróbico $\left(\mathrm{UAN}_{2}\right)$ o, umbral anaeróbico real (UANr) o, UL o, UL anaeróbico. La interpretación de estos distintos conceptos y su integración en la transición aeróbicoanaeróbico (Kindermann, Simon y Keul, 1979) provocó que autores posteriores (Faude at al., 2009) hablasen de tres grupos terminológicos. Tras encontrar 25 diferentes conceptos de umbrales (ULs), los dividieron en tres categorías. Categoría 1: autores que usan el comienzo de la acumulación de lactato fijo en sangre(OBLA) durante test incrementales. Categoría 2: otros conceptos ULs pretenden detectar el primer aumento en OBLA por encima de los niveles de referencia. Categoría 3: consiste en conceptos ULs que apuntan a detectar el MLSS. Parece que el término y significado de OBLA se establece como punto de referencia. Al respecto, Del Río Valdivia et al. (2017) indican que:

Cómo no siempre es fácil observar este punto de inflexión, se ha establecido un valor arbitrario: La intensidad del ejercicio a la cual el lactato se mantiene en un estado estable, antes de que comience a incrementarse desproporcionadamente y se acumule en la sangre $u$ OBLA. Este valor se estableció en 4 mmol de ácido láctico por litro de sangre. (del Río Valdivia et al., 2017, p.58)

Hall et al. (2016) indican que muchos conceptos erróneos continúan impregnando la evaluación deportiva. Un primer error es definir inadecuadamente los términos. Esto ha llevado a redefinirlos por lo que un mismo concepto tiene varias interpretaciones. La actual gran variedad de focos de la literatura especializada hace muy difícil la comprensión de la situación a cualquiera que no sea fisiólogo del ejercicio. Los principales focos de las publicaciones son:

-Necesidad constante de dar validez y fiabilidad a una gran cantidad de protocolos ventilatorios, incrementales de lactato, continuos; . . para la fiabilidad de las mediciones de lactato; para la determinación de ULs; para la validación de diferentes pruebas; para algoritmos basados en regresiones en la evaluación del UAN...

- Búsqueda incesante de correlaciones. Entre umbrales de lactato y ventilatorios; entre estos y el MLSS; entre el intercambio de lactato y gases en test incrementales o de estado estable; entre el UAN, el UANI y el MLSS; entre el UAN y distancias de competición; entre el UAN y el inicio de la acumulación de lactato en sangre; entre diversos índices de ULs y su relación con la velocidad de carrera de diferentes distancias; entre velocidades en cinta o cicloergómetro y predicciones de rendimiento en diferentes distancias; entre la potencia pico y el umbral de lactato en corta distancia; entre el MLSS, el umbral de compensación respiratoria y potencia crítica; entre la velocidad crítica, la velocidad del máximo estado estable y el punto de inflexión del lactato en los corredores; ente el UAN y la potencia máxima aeróbica; diferencias entre umbral de lactato en sangre arterial y sangre venosa; entre los índices usados para detectar el UAN; entre la ventilación y los ULs que siguen dietas normales, bajas y altas en carbohidratos (CH); entre el efecto de las modificaciones dietéticas en el umbral de lactato y el inicio de la acumulación de lactato en la sangre durante el ejercicio incremental...

-La reproducibilidad de diferentes protocolos, de los umbrales aeróbicos y anaeróbicos o la del umbral de lactato en sangre con otras mediciones como la de $4 \mathrm{mmol} \cdot \mathrm{L}-1$., de la Frecuencia cardíaca (FC) y la percepción.

-Los valores que se toman en las pruebas o test y como se interpretan los resultados.

Con este panorama parece imposible unificar criterios. Pero a pesar de esta gran dispersión de intereses se pueden concretar algunos aspectos. Así, se estima que el UL puede ser una herramienta útil para evaluar. Se busca correlacionarlo con OBLA, MSLL, UANI o alguna de sus fases. Ganan terreno los protocolos que utilizan el UL como predictor del rendimiento o de rendimiento en MSLL mostrando buenas correlaciones (Santos-Concejero et al. 2013;Wahl, Zwingmann, Manunzio, Wolf y Bloch, 2018). La potencia en el UL y, el $\mathrm{VO}_{2}$ en el UL son un fuerte predictor de rendimiento en ciclismo, aunque no tanto como el peso y los años de carrera experiencia.

Según Svedahl y Macintosh (2003) la prueba ideal debe producir de manera consistente una intensidad de ejercicio cercana al MLSS, que consideran el mejor predictor del UAN. Este es la intensidad del ejercicio en el UAN. Así, aunque el $\mathrm{VO}_{2 \text { máx }}$ sea similar entre grupos de profesionales y amateurs, los primeros son capaces de sostener cargas de trabajo mucho mayores en su MLSS. Estas predicen fuertemente el rendimiento. Hall et al. (2016) muestran que, en corredores de élite, la velocidad de carrera en MLSS suele mejorar el rendimiento. El $\mathrm{VO}_{2}$ y la velocidad de carrera en el MLSS son un buen predictor del rendimiento. Pero para predecir con precisión el MLSS o FC en ciclistas profesionales hay que verificarlo mediante una prueba de carga constante más larga (30 minutos). Las mediciones de fiabilidad en test-retest incrementales en cinta rodante al MLSS son pobres. Su valor absoluto tampoco predice el rendimiento en resistencia. Un sujeto con un MLSS a 4 $\mathrm{mmol} \cdot \mathrm{L}-1$ no es mejor fondista que otro que lo tenga en $2 \mathrm{mmol} \cdot \mathrm{L} 1$. Van Schuylenbergh, Vanden Eynde y Hespel (2004) encontraron poca correlación entre el MLSS de potencia o FC y la validez de diferentes métodos de medición de umbrales de lactato y ventilatorios.

OBLA. Partiendo de su valor de inicio, otra tendencia establece zonas basadas en los umbrales (González Fimbres, Griego Amaya, Cuevas Castro y Hernández Cruz, 2016). Es un enfoque de la estimación del UAN que asume que tiene una concentración absoluta de lactato en sangre de $4 \mathrm{mmol} \cdot \mathrm{L}-1$ en un ejercicio incremental, pero, no tiene en cuenta la variabilidad individual. Por ello las pruebas de UANI son más efectivas pero complejas. Evitan algunas de las deficiencias en OBLA. En cualquier caso, el porcentaje de OBLA o MLSS es confiable para normalizar la intensidad del ejercicio.

La relación entre UANI y MLSS es algo variable. Parece que el UANI se produce con una carga de trabajo mayor que el MLSS y que puede ser una estimación confiable del rango de MLSS. El método de identificación del punto de inflexión de lactato para OBLA o MLSS 
puede ser la fuente de dificultad en la reproducción en los resultados de la prueba de UL. Sin embargo, Zúñiga, Housh, Camic, Bergstrom, Schmidt, y Johnson (2014) demostraron que los métodos de análisis de la curva de lactato en sangre no son importantes, pero sí observaron que las diferencias en los protocolos de prueba tuvieron grandes efectos en la estimación de OBLA y MLSS.

Finalmente, los avances tecnológicos tienen cada vez mayor validez, fiabilidad y reproducibilidad. Los métodos de campo para controlar la intensidad del ETO con potenciómetros o GPS son cada vez más exactos. Son herramientas útiles. A eso se une la búsqueda de pruebas que no generen los problemas descritos y ello hace que, por ejemplo, en ciclismo, la potencia máxima de 1 hora o el test de 20 min sean un fuerte predictor de rendimiento o, que la velocidad máxima sostenible en una cinta rodante prediga el rendimiento de carrera de $5000 \mathrm{~m}$ mejor que el $\mathrm{VO}_{2 \text { máx }}$ la economía de carrera, el $\mathrm{VO}_{2}$ en el umbral de lactato, el OBLA o, el MLSS. La potencia y la velocidad crítica han demostrado tener buena correlación con la potencia y la velocidad en el MLSS y OBLA Por ello, muchos fondistas están pasando de planificaciones basadas en pruebas de lactato a planes con ZETO basados en el ritmo aeróbico máximo o crítico o, la potencia de umbral funcional. Mirando al futuro, hay autores como Seiler que están prediciendo que estas últimas medidas funcionales de rendimiento al alcance del deportista son más fáciles de reproducir. En caso de acudir a un laboratorio, el entrenador y deportista deben informarse bien del protocolo a aplicar, qué y cómo se le va a medir para poder reproducirlo posteriormente.

\section{Umbral Anaeróbico.Aspectos didácticos.}

Concentración del lactato. Varía según el punto de la extracción, así como el método de toma de muestras de sangre (Robergs et al.,1990; Coyle, 1985; Roecker, Schotte, Niess, Horstmann y Dickhuth, 1998; Feliu, et al., 1999) y, sobre todo, puede variar ampliamente, como resultado de la ingestión de $\mathrm{CH}$ previa al test. El agotamiento de las reservas de glucógeno por una dieta baja en $\mathrm{CH}$ o, el ejercicio exhaustivo previo bajaría la concentración en comparación con una ingesta normal. Esto puede llevar a un menor desplazamiento de la curva de lactato y no se puede interpretar como una mejora en la capacidad de resistencia. Otros factores como la composición de la fibra muscular, la actividad enzimática lipolítica, glucolítica, la densidad capilar o mitocondrial podrían influir en las curvas de lactato en sangre.

Métodos simples de determinación del UAN. Identificar el punto de deflexión de la FC puede ser útil para prescribir la intensidad del ejercicio (Semin, Stahlnecker, Heelan, Brown, Shaw, B., y Shaw, 2008), por lo que determinar el UAN a partir de la FC es el método más popular. Diversos estudios indican que la ecuación de Tanaka para calcular la FC máxima teórica, es la que muestra mayor índice de exactitud, en hombres y mujeres jóvenes, pero, se siguen utilizando otras fórmulas inexactas con desajustes severos en las ZETO (Bouzas Marins, Ottoline Marinsa y Delgado Fernández, 2010; Carbonell Martínez, Ferrándiz Moreno y Pascual Verdú; 2017). De fácil aplicación práctica fue el hallazgo de Skiner y McLellan (1980) encontrando que el UAN estaba entre el 90-94\% del valor de la frecuencia cardíaca máxima (FC ${ }_{\text {máx }}$ )

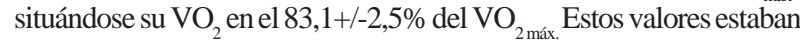
entre el valor seis y siete en los puntos de una escala de percepción subjetiva del esfuerzo (PSE) de cero a 10. Serrano Pérez (2015) indica que las pruebas PSE, son cada vez más tenidas en cuenta como complementos de información e incluso como sustitutos de los ritmos o FC. Murillo Lorente, Álvarez Medina y Manomelles Marqueta (2016) muestran que se puede predecir la FC a través de la PSE siendo una forma rápida y eficaz de cuantificar la carga interna sin necesidad de pruebas complejas. Seiler y Kjerland (2006) no encontraron diferencias en la cuantificación de la intensidad entre la FC y el PSE en atletas de alto nivel, pero Høydal y Nord (2017) reportan en jóvenes que subestima la intensidad en el UL y da como resultado una distancia recorrida menor. Por ello aconsejan el uso de monitores de FC frente a la sensación subjetiva.

Las pruebas del «habla» están despertando interés (Rodríguez-
Marroyo, Villa, García López, y Foster, 2013). Las pruebas de potencia/velocidad crítica o de velocidad aeróbica máxima (Lopes-Motoyama, et al., 2013), son test de ritmo o velocidad que han ido sustituyendo a otros como el de Conconi (aparecido en 1982). Se determinan las zonas de ritmos (con toma o no de lactato) y se pueden complementar con la FC correspondiente a cada ritmo. Boudet, Garet, Bedu, Albuisson y Chamoux (2002) estimaron que durante ejercicios de alta intensidad puede ser mejor prescribir la intensidad de carrera en base a la velocidad (ritmo) que en base a la FC. De Lucas et al. (2012) describen que no hay diferencias estadísticas significativas entre un test de velocidad crítica y uno al MLSS

Test de laboratorio y UAN. Las pruebas de laboratorio presentan problemas varios. Diversos autores demostraron que existen diferencias significativas en las mediciones de $\mathrm{FC}_{\text {máx }}$ cuando estas se realizan en laboratorio frente al ETO o competición. Dependiendo de la prueba y del laboratorio utilizado, hay diferencias entre test de campo en fondistas competiendo (206 \pm 4latidos $\cdot \mathrm{min}^{-1}$ ) o entrenando (207 \pm 5 .0 latidos $\cdot \mathrm{min}^{-}$ $\left.{ }^{1}\right)$ frente a los test de laboratorio (194 \pm 2 latidos $\cdot \mathrm{min}^{-1}$ ) (Boudet et al., 2002; Jeukendrup y Diemen, 1998; Kunduracioglu, Guner, Ulkar y Erdogan, 2007; Lambert, Mbambo y Gibson, 1998; Semin et al., 2008). Incluso se ha reportado en otros deportes (Coutinho, Watson, Brickson, y Sanfilippo, 2017). Se ha observado, durante test de campo, que la concentración sanguínea de lactato, medida a una FC dada, es mayor que la medida durante un test de laboratorio (Vergès, Flore y FavreJuvin, 2003). La menor intensidad de eto basada en la medición de la FC en laboratorio frente al eto o competición, podría reducir las adaptaciones y el rendimiento (Potteiger y Weber, 1994). Incluso simular una competición demostró provocar un incremento mayor en el $\mathrm{VO}_{2}$ y en la FC en comparación con el incremento provocado en laboratorio (Foster, Green, Snyder y Thompson, 1993).

UAN como factor limitante en el eto de fondo. El factor limitante del rendimiento en fondistas ha sido considerado, tradicionalmente, el $\mathrm{VO}_{2}$. Depende de la dotación genética, edad, peso, sexo y nivel de ETO. El porcentaje de mejora en la temporada no es alto; sobre el $6 \%$ del $\mathrm{VO}_{2}$ máx (Carazo Vargas y Moncada Jiménez, 2015). Depende del nivel del deportista, del deporte y del momento de la temporada. Disminuye a partir de los 25 años hecho importante que no explicaría que haya fondistas de más de 30 años en lo más alto. Los valores del $\mathrm{VO}_{2}$, se estancan en pocas semanas, aunque la capacidad de rendimiento siga mejorando. A la vez Acevedo y Goldfarb (1989) apreciaron desplazamientos hacia la derecha en el perfil del lactato sanguíneo, es decir, de mejora en el UAN. El VO no es el factor limitante ni un buen predictor de por sí. El $\mathrm{VO}_{2 \text { máx }}$ es una medida del potencial máximo de un atleta, mientras que el $\mathrm{VO}_{2}$ en el UL o el ritmo/potencia en el UL es una medida de la capacidad actual del atleta. La resistencia no está determinada por la cantidad de oxígeno aportado por los músculos durante el trabajo prolongado intensivo, si no por la adaptación de los músculos, de utilizar este oxígeno (Versohansky, 2007).

Calderón et al. (2008) señalan que «el fondista intenta llevar una intensidad de ejercicio lo más próxima a su $\mathrm{VO}_{2 \text { máx }}$ intentando aguantar el máximo tiempo posible» (p.323). A lo largo de los años ha adquirido gran importancia conocer cuánto tiempo se puede sostener un ejercicio lo más próximo al $\mathrm{VO}_{2 \text { máx }}$. El ganador de un maratón no puede predecirse midiendo su $\mathrm{VO}_{2}$ en laboratorio, ya que un buen rendimiento supone algo más que un $\mathrm{VO}_{2 \text { max }}$ elevado. El maratoniano Alberto Salazar, ex recordman mundial, tenía su $\mathrm{VO}_{2 \text { máx }}$ sólo en $70 \mathrm{ml} / \mathrm{kg}$, pero era capaz de correr al 86\% del mismo, muy por encima de otros con mejor $\mathrm{VO}_{2 \text { máx }}$ (ver ejemplo de tabla l). Santos-Concejero et al. (2013) señalaban quela capacidad de correr sin acumular lactato es más importante que la absorción máxima de oxígeno o el coste energético de correr (Cr). Verkhoshansky (2007) relata que para poder competir cerca del $\mathrm{VO}_{2 \max }$ (UAN), es decisivo el componente periférico de extracción de oxígeno y que el momento en el cual empieza la actividad de las fibras de contracción rápida es el de la llegada al UAN siendo explícito al indicar:

Cuando el nivel de resistencia aumenta de tres a cinco veces, la cantidad de mitocondrias y la capacidad oxidatíva en el músculo esquelético, aumentan dos veces [50\%], mientras que el $\mathrm{VO}_{2 \text { máx }}$ aumenta sólo 
10-14\%. El mayor nivel de intensidad que podría alcanzarse durante el ejercicio prolongado sin una acumulación de lactato en sangre considerable es un mejor indicador de la capacidad de resistencia que, el $\mathrm{VO}_{2 \text { máx }}$ (Verkhoshansky,2007, p.2)

Tabla 1. Efecto en el rendimiento de la ubicación del UAN con respeto a diferentes valores de $\mathrm{VO}_{2 \max }$ en nadadores. Adaptado de Maglisco.

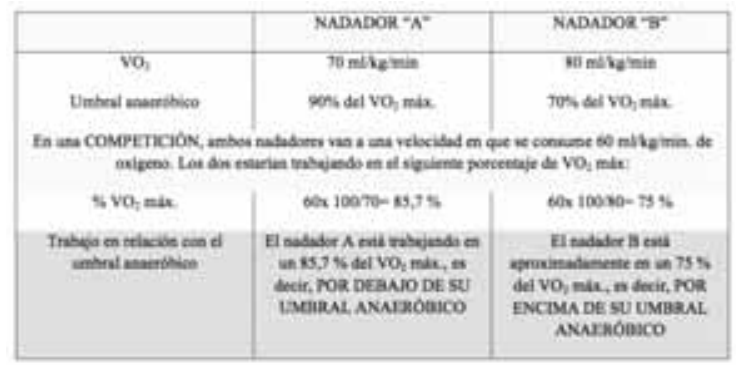

Tabla 2. Vías o zonas de eto propuesta por los autores. Elaboración propia.

\begin{tabular}{|l|}
\hline -AEROBICO LIGERO LIGERO; ALL \\
\hline - AEROBICO LIGERO MEDIO; ALMED \\
\hline - AEROBICO LIGERO INTENSO; ALINT \\
\hline - UMBRAL AEROBICO; UA \\
\hline - SUB UMBRAL ANAEROBICO 1 ; SUBUANI \\
\hline - SUB UMBRAL ANAEROBICO 2; SUBUAN2 \\
\hline - SUB UMBRAL ANAERÓBICO 3; SUBUAN3 \\
\hline - UMBRAL ANAEROBICO; UAN \\
\hline - MAXIMO CONSUMO OXIGENO; $\mathrm{VO}_{2}$ máx \\
\hline - RESISTENCLA ANAEROBICA; RAN \\
\hline
\end{tabular}

Zonas de entrenamiento. Las investigaciones suelen distribuir, con marcadores biológicos, las cargas de ETO en tres zonas (1 a 3). Los informes de pruebas de esfuerzo tienen entre tres y cinco zonas. Actualmente los diseños de los entrenadores presentan de cinco a siete zonas de diferentes autores. A nivel práctico y didáctico diferentes autores prefieren un alto número de zonas porque cierran mejor el rango de trabajo, dando también, más juego o posibilidades de variación como las propuestas en la Tabla 2.

Entrenar el UAN. Los resultados deportivos están directamente relacionados con el control de las intensidades de trabajo (SergeyevichMishchenko y Dmitriyevich-Monogarov, 2001). A mayor distancia, más importante es la relación entre control de la intensidad y rendimiento. Estos autores indican que, en maratón, el resultado está muy estrechamente vinculado con el UL $(r=0,98)$. La correlación más alta entre la velocidad de carrera con la FC del UL y el resultado de las competiciones está en carreras de una hora de duración $(\mathrm{r}=0,99)$. $\mathrm{El} \mathrm{VO}_{2 \text { máx }}$ durante el ejercicio y el UAN se pueden mantener unos 60 minutos (Billat et al. 2000) entre el 60 y el 80\% del $\mathrm{VO}_{2 \text { máx }}$ (Lajoie, Laurence y Trudeau, 2000). Por debajo del UAN, el ejercicio puede mantenerse durante varias horas. Por ello, la FC media, el ritmo, la velocidad o potencia durante una competición de una hora corresponderá al valor UAN. Santos Concejero et al. (2013) encontraron una correlación lineal significativa entre la velocidad de OBLA y la de tiempos en $10 \mathrm{~km}(r=$ $0.761, P<0.05)$ y tiempos en $3 \mathrm{~km}(r=0.561, P<0.05)$. Las pruebas de intensidad constante deben durar al menos 20 minutos, aunque las pruebas que duran 30 minutos o más se han utilizado con más frecuencia (Svedahl y Macintosh, 2003). En natación se utiliza la prueba de 30 min (Olbrecht, Madsen, Mader, Liesen y Hollmann, 1985) dando una velocidad media de nado con una correlación elevada y significativa ( $\mathrm{r}=$ 82) a la velocidad OBLA o, en ciclismo, el actual test de 20 minutos (Allen y Coggan, 2006). Teniendo en cuenta el valor medio y máximo de esa frecuencia (ritmo medio, vatios medios...) se crean las tablas de ZETO. Por esto, las distancias o tiempos largos de trabajo son los más recomendables.

El UAN es el paso más rápido que un atleta puede mantener durante un período de tiempo extendido sin acumular cantidades adicionales de lactato (Calderón et al., 2008; Sergeyevich-Mishchenko y Dmitriyevich-Monogarov, 2001; Santos Concejero et al., 2013). El descanso en distancias largas es grande. Esto responde más a una necesidad motivacional asociada a la intensidad y duración de las siguientes repeticiones que, al hecho de evitar que baje el pulso entre repeticiones como cuando se usan distancias cortas. Estas son menos recomendables porque están más determinadas por la adecuada relación de densidad (trabajo/descanso) siendo más importante cuanto más cortas sean las distancias. El uso de estas distancias cortas es casi específico de natación (25, 50, 100 y 200 m).

El UAN en la planificación. Diferentes estudios (Guro, Tønnessen y Sandbakk, 2017; Seiler y Kjerland, 2006; Seiler y Tønnessen, 2009; Stöggl y Sperlich; 2014) muestran que, contra lo que parece y los modelos de alta intensidad reflejan, la elite mundial (podios mundiales y olímpicos en deportes de resistencia) tiende a polarizar las cargas utilizando mayoritariamente en la temporada las ZETO más suaves (zona 1) y sólo muy cerca de la competición principal (raramente más de dos al año) las cargas duras de UAN y $\mathrm{VO}_{2 \text { max }}$ (zona 2 y zona 3 o HIT-HIIT) pero sin apenas entrenar la zona UAN (zona 2). De hallazgo importantísimo para el ETO es observar que de entre estas tres vías, la que menos se entrena es, precisamente la del UAN (Ingham, Fudge y Pringle, 2012; Seiler y Kjerland, 2006; Stoggl y Sperlich, 2015; Tjelta, 2013; Tonnessen, et al., 2014). No se trabaja en todos los mesociclos del año a pesar de que famosos modelos (ATR...) lo permiten. Manunzio, Mester, Kaiser y Wahl, (2016) muestran que una planificación basada en zona 2 o UAN (39\% del total del año) reporta sólo incrementos moderados en los parámetros fisiológicos. Por ello los mejores tienden a planificar a través de periodizaciones clásicas frente a otros modelos (Guro et al. 2017).

Las investigaciones de la escuela noruega sobre la distribución anual de las cargas en los mejores fondistas mundiales de diferentes deportes de resistencia enseñan que los diseños polarizados son los más eficientes. El trabajo pionero de Seiler y Tønnessen (2009) analizó 15000 sesiones de eto registradas, de tres campeonas mundiales (carrera de fondo, esquí de fondo y orientación). En ellas, aproximadamente el 85 $\%$ de sus sesiones de ETO fueron realizadas como esfuerzos continuos de intensidad baja a moderada (lactato sanguíneo d»2 mm) y el resto (15\%) en o por encima. Proporciones muy similares se repiten en estudios posteriores con podios mundiales y olímpicos. Por ello se empezó a hablar de la regla 80-20 como modelo de distribución de las cargas en la planificación.

El estudio de Guro, Tønnessen y Sandbakk (2017) realizó un análisis de 8105 sesiones de ETO entre los 20 y 35 años de la mejor esquiadora de la historia. Había tenido grandes éxitos mundiales de los 20 a los 27 años, pero obtuvo más oros cuando pasó, de los 28 a los 35 años, de la ya excelente proporción de las cargas polarizadas del 88\% en zona $1,2 \%$ en zona 2 y $10 \%$ en zona 3 a una proporción de 92/3/5. Se concluyó que el éxito estaba en el patrón polarizado con una gran cantidad de trabajo en zona $\mathrm{UL}_{1}$ (Zona 1 , d»2mM de lactato sanguíneo, 60-82\% de la máxima FC) alcanzando los mejores resultados de la historia del esquí femenino. Estudios con fondistas en podios mundiales realizados por la escuela noruega, entre $2006 \mathrm{y}$ la actualidad, confirman rangos de trabajo similares a los descritos. Se trata de hallazgos sorprendentes muy a tener en cuenta porque, además, en el historial de vida deportiva interanual de estos deportistas la proporción del trabajo realizado en zona 1 se mantiene siempre como la más alta con diferencia. Esto es, sin duda, un hallazgo que es necesario transferir al conocimiento del entrenador cotidiano, más con la moda HIT de por medio.

UAN y periodización clásica. La periodización consta de periodo preparatorio, competitivo y de transición. Este esquema se puede suceder una, dos o tres veces al año (figura 1 ).

Según lo descrito por la escuela noruega, el momento de iniciar el eto UAN podría ser el periodo precompetitivo. Atendiendo a las ZETO señaladas en la tabla 2, en este período se colocarían cargas de forma progresiva entre SUBUAN y SUBUAN $_{3}$ hasta que, llegado el período competitivo, se introducirían cargas también progresivas en el mismo UAN (por ejemplo, de menor a mayor duración o en relación a la densidad de trabajo). La tabla 3 muestra cómo se podrían distribuir las diferentes zonas de eto en una periodización anual.

* ZETO = Zonas de eto; $\mathrm{PPG}=$ Período preparatorio general; PPE = período preparatorio especial; $\mathrm{PPREC}=$ período precompetitivo; $\mathrm{PC}$ = período competitivo; $\mathrm{s}$ = sistemáticamente; $\mathrm{pfp}$ = puntualmente 

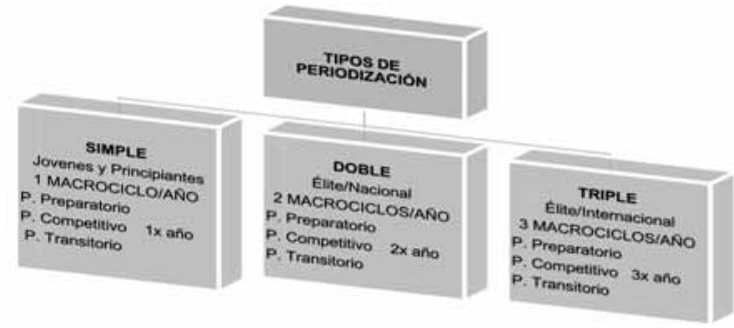

Tabla 3.

Ejemplo práctico de distribución de las zonas de eto en la periodización tradicional. Elaboración propia.

\begin{tabular}{|c|c|c|c|c|}
\hline ZETO & PPG & PPE & PPREC & PC \\
\hline ALL & SI (s) & SI (cr) & $\mathrm{Si}$ (cr) & Si (cr) \\
\hline ALMD & SI (s) & SI (s) & Si (cr) & Si (cr) \\
\hline ALINT & SI (s) & SI (s) & SI (s) & Si (cr) \\
\hline UA & SI(s) & SI (s) & SI(s) & SI, (p) \\
\hline SUBUAN1 & SI (pfp) & SI(s) & SI, (s) & SI, (p) \\
\hline SUBUAN2 & SI (cc) & SI (pfp) & SI, (s) & SI, (s) \\
\hline SUBUAN3 & NO & SI (cc) & SI, (pfp) & SI, (s) \\
\hline UAN & NO & No & SI, (cc) & SI, (sfp) \\
\hline VO2max & NO & NO & SI, (pfs) & SI, (sfp) \\
\hline RAN & NO & NO & SI, (pfs) & SI, (sfp) \\
\hline
\end{tabular}

al final del período; cc = competición controlada; $\mathrm{cr}$ = como regenerativo; $\mathrm{p}=$ puntualmente; $\mathrm{pfs}=$ puntualmente $\mathrm{y}$ al final del período sistemáticamente; $\mathrm{sfp}=$ sistemáticamente de forma progresiva.

UAN en el período pre y competitivo ¿Cuántas veces por semana? El UAN es un ritmo de gran desgaste (Londeree, 1997). En la semana de competición de no hace falta meter más de una sesión UAN (Billat, Demarle, Slawinski, Paiva, y Koralsztein, 2001) sea ETO o competición. Más, podría ser un riesgo (Chwalbinska-Moneta et al., 1998). En el precompetitivo, habría que empezar en las vías anteriores (SUBUAN - $_{1}$ SUBUAN $_{3}$ con ramalazos en el propio UAN, sólo, en las competiciones de este período. Si se hacen a ritmo $\mathrm{SUBUAN}_{2}-\mathrm{SUBUAN}_{3}$ podrían ser, más cortas en duración que la distancia de competición y, más largas si se hacen en $\mathrm{SUBUAN}_{1-2}$. En el competitivo, la sesión podría ser más corta del tiempo/distancia de competición o, similar en duración y ritmo, si se modula en repeticiones cortas, para asimilarla mejor, de forma progresiva en intensidad y duración en las semanas. Además, en este período, hay que introducir otras cargas altas en relación al $\mathrm{VO}_{2}$ (HIT) o resistencia anaeróbica (RAN). Si se opta por vías justo por debajo del UAN (SUBUAN ${ }_{3}$ ) podrían ser con distancias más largas que las de competición al ser más asequibles, aunque también exigentes.

Modelos de ciclización compleja. Surgen del modelo de Tschienine en 1984. Buscaban la adaptación a más y mayores cargas durante el año, para pasar así a un supuesto nivel superior de prestación Se basa en modelos de cargas acentuadas o concentradas. Respondían a la necesi-

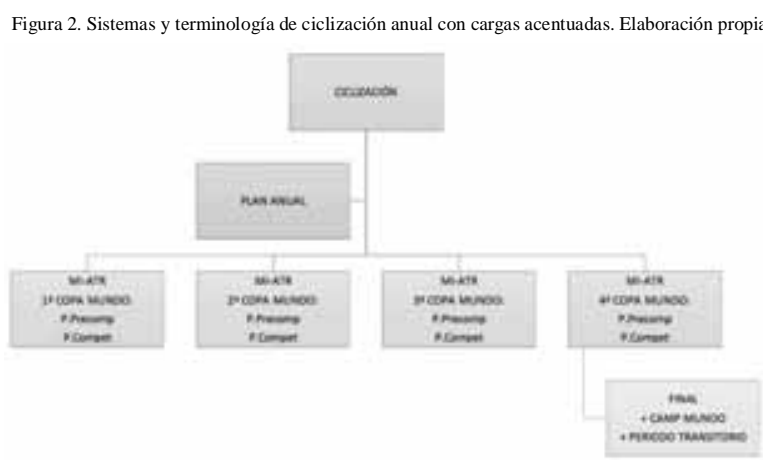

Figura 3. Sistema de macrociclos integrados. Adaptado de Navarro et al. (1990).

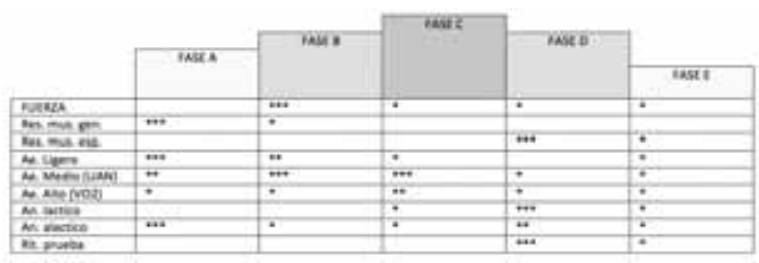

dad de competir al máximo nivel toda la temporada (figura 2). La duración de los ciclos puede variar. Es el caso de los ATR (asimilación, transformación, realización) de Issurin (2010) o de los macrociclos integrados (MI) de Navarro, Arellano, Carnero y Gosálvez, (1990). En ambos, hay que resolver el problema de sostener la mayor carga posible durante el mayor tiempo posible, lo que supone un enorme desgaste al organismo.

En los macrociclos integrados (MI) la distribución de las ZETO se ve en la figura 3 (MI de 12 semanas). Este esquema se puede repetir cuantas veces se necesite. Cada fase representa dos semanas. ElETO de UAN sería el referido al «aeróbico medio» y, como se observa, tendría su mayor carga de trabajo sobre todo en la segunda y tercera semana coincidiendo con un aumento del volumen. Disminuye su trabajo en las semanas siete-ocho y, en las dos últimas, queda a modo de mantenimiento. Este modelo es totalmente contrario a como se aplica el UAN en la polarización.

El sistema ATR de Issurin (2010) representa las fases de asimilación-transformación-realización y la distribución es como muestra la figura 4 .

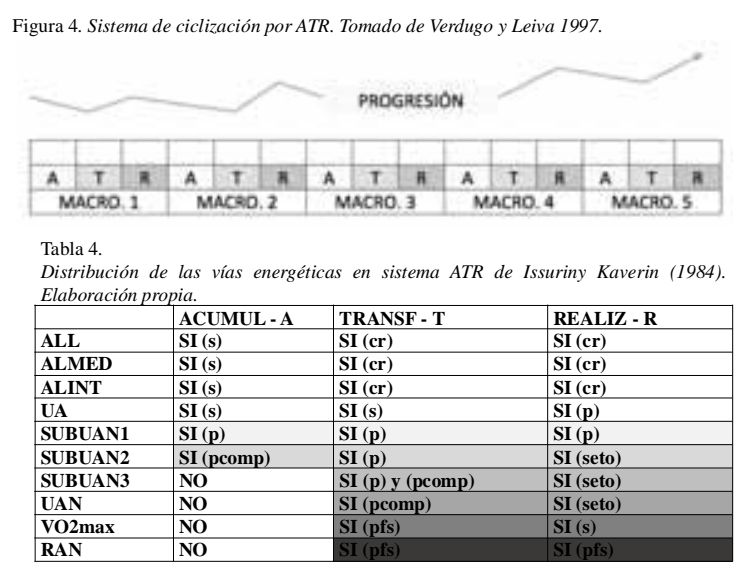

El gráfico representa los ciclos ATR a lo largo de un año para cinco grandes competiciones. El ETO de UAN se incorpora sólo en las fases «R» del año (tabla 4). Se entrenaría tantas veces al año como fases $\mathrm{R}$ haya en el mismo. Tanto en el caso del MI como de los ATR la aplicación por semana del número de sesiones debería ser similar a la señalada en la periodización. Como ocurre con los MI, la suma de semanas de trabajo duro, en las fases $\mathrm{R}$, supera con creces las que se realizarían en las planificaciones por polarización.

*ACUMUL = Acumulación; TRANSF = transformación; REALZ = realización; $\mathrm{PC}=$ período competitivo; $\mathrm{s}=$ sistemáticamente; $\mathrm{p}=$ puntualmente; pcomp = puntualmente en competición controlada; pfp = puntualmente al final del período; $\mathrm{cc}$ = competición controlada; $\mathrm{cr}$ = como regenerativo; seto = sistemáticamente en entreno; pfs = puntualmente y al final del período sistemáticamente; $\mathrm{sfp}$ = sistemáticamente de forma progresiva.

El UAN en la planificación plurianual. Polischuk (1993 citado en Platonov, 2001) dispuso un sistema cuatrienal (ciclo olímpico) donde cada año prioriza ZETO de diferente intensidad (tabla 5). Se observa que el año donde más se trabajan las zonas duras (UAN-VO-RAN) es el cuarto año u olímpico (18²\%) mientras que en el primero se trabajarían las mismas zonas el 12’3\%.

Tabla 5.

Distribución de la carga (pulso) del trabajo en un ciclo olímpico. Adaptado de Platonov (2001)

\begin{tabular}{|l|l|l|l|l|}
\hline INTENSIDAD & AÑ̃ I & AÑ̃ II & AÑ̃ III & AÑ̃ IV \\
\hline hasta 130pm & $27^{\prime} 4 \%$ & $25^{\prime} 2 \%$ & $23^{\prime} 3 \%$ & $23 \%$ \\
\hline $130-150 p m$ & $29^{\prime} 6 \%$ & $29 \%$ & $27^{\prime} 5 \%$ & $28^{\prime} 8 \%$ \\
\hline $150-170 p m$ & $30^{\prime} 7 \%$ & $32^{\prime} 7 \%$ & $34^{\prime} 8 \%$ & $34 \%$ \\
\hline$+170 p m$ & $12^{\prime} 3 \%$ & $13^{\prime} 1 \%$ & $14^{\prime} 5 \%$ & $18^{\prime} 2 \%$ \\
\hline
\end{tabular}

* pm = pulsaciones minuto (trabajo)

A pesar de las supuestas ventajas de los sistemas de ciclización tipo ATR-MI, parece que los mejores fondistas apenas las utilizan (Billat et 
al., 2001; Schumachery Mueller, 2002; Seilery Kjerland, 2006; Steinacker et al., 1998). Esteve-Lanao, Foster, Seiler y Lucia, (2007) observaron que, entre varias posibles, la distribución $80 \%$ en zona uno (UA o $\mathrm{UL}_{1}$ ), el 5-12 \% en zona dos (UAN o $\mathrm{UL}_{2}$ ) y el 8-15\% en zona tres $\left(\mathrm{VO}_{2 \operatorname{máx}}-\mathrm{HIT}\right)$ era la que mejores resultados daba. La elite mundial realiza la mayor parte de su volumen anual en zonas más bien suaves. Se entrena menos de lo esperado en la zona dos de UANy zona tres de $V O_{2 \max } y R A N$. Se dedica más tiempo al trabajo de $V O_{2 \text { máx. }}$ que al UAN tratándose, sin embargo, de una vía más dura (Tjelta, 2013; Tønnessen et al., 2015; Tønnessen et al., 2014). Esto es un factor clave a tener en cuenta en el diseño de entrenamientos (Seiler y Tønnessen, 2009) y demuestra:

$1^{\text {a }}$. Que ese porcentaje de UAN (5-12\%) es insuficiente para tener una distribución homogénea del mismo, a lo largo de los diferentes mesociclos de la temporada.

$2^{\circ}$. Por ello, lo que se hace es entrenarlo de forma polarizado (POL), concentrado en momentos determinados de la temporada.

\section{Discusión}

La heterogeneidad de términos usados en relación a los umbrales ahonda en su incomprensión y es un elemento distorsionador en la aplicación práctica de los ETOS. Es necesario que algún organismo reconocido proponga un diccionario de términos y un estándar de protocolos en laboratorio. Se ha pasado terminológicamente por muchos términos. El más actual, UL, no se relaciona con el UA ni UAN sino con el valor previo del primer aumento considerable de concentración de lactato. López Chicharro y Vicente Campos (2017) lo definen como el valor justo anterior a la toma donde se dispare el lactato por primera vez en una prueba de esfuerzo, siempre que sea superior a $0{ }^{\prime} 5 \mathrm{mmol} \cdot \mathrm{L}-1$ sobre ese valor previo. Es decir, el valor del UL es un valor realmente bajo que normalmente no llegaría a $1 \mathrm{mmol} \cdot \mathrm{L}-1$ que en la figura 5 correspondería a $0,9 \mathrm{mmol} \cdot \mathrm{L}-1$ a la velocidad de $16 \mathrm{~km} \mathrm{~h}^{-1}$.

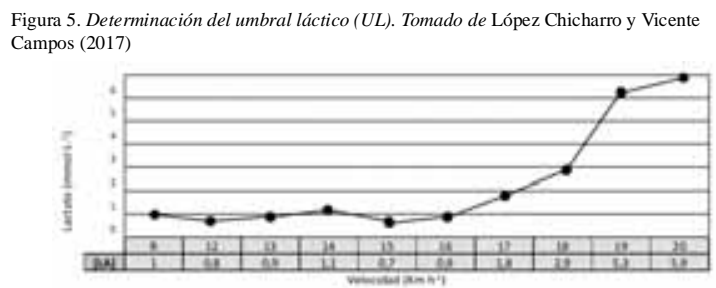

Los menores valores en FC obtenidos en laboratorio frente al ETO o competición, podrían reducir las adaptaciones y el rendimiento (Potteiger y Weber, 1994). Si las pruebas de laboratorio subestiman los valores de FC, no sería congruente utilizarlos para entrenar. Se abre un debate donde parece que cada vez más entrenadores acuden a este tipo de pruebas lo justo y necesario.

Pese a la moda HIT impuesta los últimos años parece ser que los grandes campeones no entrenan de esta manera ¿Por qué entrenan menos en las zonas duras (zona 2 y 3 ) de lo que se pensaba? Multitud de trabajos muestran el estancamiento de los niveles de rendimiento al trabajar el $\mathrm{VO}_{2 \text { máx }} \mathrm{O}$, HIT o, HIIT más de tres semanas consecutivas con varias sesiones semanales (2-3 sesiones). Resultados parecidos se han encontrado en diseños similares de UAN. Calderón et al. (2008) indicaron que, en atletas muy entrenados, el UAN:

Es tan poco modificable como el $\mathrm{VO}_{2 \operatorname{máx}}$. La bibliografía que describe las modificaciones del $\mathrm{VO}_{2 \text { máx }}$ durante el proceso de entrenamiento es abundante, no así los estudios encaminados a conocer el desplazamiento del UAN en temporada. Se observa que cuanto más entrenada es la población objeto de estudio, menor es el desplazamiento del UAN. (p.331)

Chwalbinska-Moneta et al. (1998) y Londeree (1997) señalan que es posible que, en los atletas altamente entrenados, las series repetidas de ETO en el UAN pudieran generar estrés simpático injustificado y no ofrecer ningún estímulo adicional para la mejora del rendimiento. Stöggl y Sperlich (2014) no observaron mejoras en atletas de élite en respuesta a microciclos con varias sesiones de UAN. Esteve-Lanao et al. (2007) y Guellich y Seiler (2010) sugieren que, en los atletas altamente entrenados, el tiempo de ETO cercano al UAN puede ser ineficaz, o incluso contraproducente. Estos estudios se hicieron con programas de varias semanas con dos/tres sesiones semanales de UAN. Es comprensible que ciclos con tantas sesiones en esta zona puedan afectar al deportista negativamente en su rendimiento. Por ello se indicaba que, entrenar en el UAN es muy duro y que en período pre y competitivo, una sesión sería una carga más que suficiente para desarrollarla-mantenerla. Esto explicaría que en los postest de los estudios no suela haber mejora. Contra estos ciclos con predominancia del ETO en UAN, Neal et al. (2013) demostraron que seis semanas de ciclismo de ETO polarizado produjeron en atletas altamente entrenados, mayores adaptaciones en comparación con el ETO casi exclusivo de UAN durante ese período.

¿Por qué, muchos entrenadores siguen pensando que el trabajo interválico es la única forma de optimizar el rendimiento ¿En que se basan? Uno de los problemas es que los entrenadores del día a día, no están usando los datos que los investigadores noruegos han ido mostrando. Es fácil reportar casos donde se usa el HIT inadecuadamente ¡Sencillamente se abusa de la intensidad! El HIT es beneficioso entre 38 semanas, los estudios lo confirman... pero los deportistas que no cumplen la regla 80-20 parece que no son podios mundiales. La investigación de las supuestas bondades HIT empezó y se prolongó en el ámbito del estudio de sujetos sedentarios u obesos. Demostraban que unas semanas de trabajo mejoraban parámetros fisiológicos y el rendimiento de resistencia en personas desentrenadas o moderadamente activas (Daussin et al., 2007; Denis et al., 1984; Gaskill et al, 2001; Londeree, 1997; Talanian et al., 2007) de lo que se aprovechó el marketing del mundo del fitness. Durante años se ha querido trasladar las bondades de estos resultados a deportistas competitivos que ya los aplicaban en sus ciclos. Fue, a tenor de las investigaciones indicadas, un error.

Cuando la clase mundial entrena de forma polarizada en zona 2, se reportan mejoras en las carreras o test hechos a ritmos de UAN. Sandbakk, Holmberg, Leirdal y Ettema (2011) mostraron dentro de un esquema polarizado que esquiadores de sprint de fondo de clase mundial de Noruega presentaban un volumen de eto mayor, cerca de la zona deUAN en comparación con los esquiadores denivel nacional. Evertsen, Medbo y Bonen (2001) demostraron que en los esquiadores de fondo deélite se observaron mejoras en la velocidad de carrera en el UL y en el rendimiento, en una carrera de 20 min cuando entrenaban a una intensidad que producía 3-4 mmol- $\mathrm{L}^{-1}$ de lactato en comparación con una intensidad de eto menor $\left(<3-4 \mathrm{mmol} . \mathrm{L}^{-1}\right)$.

\section{Conclusiones}

Actualmente no está resuelto el problema terminológico/conceptual. Existe una gran dispersión terminológica en relación a los ULs de ETO que es necesario abordar, clarificar y simplificar en los foros pertinentes. Hay que determinar la forma más eficiente de medir el UAN. Los métodos más accesibles son los que relacionan FC e intensidad. Los de velocidad aeróbica máxima o crítica parece que son los más fiables y reproducibles, estableciendo los ritmos de las ZETO en base a ello. Cada vez son más usados aparatos como potenciómetros o GPS y pruebas de PSE.

En las pruebas de laboratorio la $\mathrm{FC}_{\text {máx }}$ medida en laboratorio puede no ser una elección óptima para diseñar un programa de ETO. El hallazgo de Skiner y McLellan (1980) fue y es una forma muy simple de obtener el valor en FC del UAN para establecer las zonas de ETO (el UAN esta entre el 90-94\% del valor de la $\mathrm{FC}_{\text {máx }}$ del deportista). En laboratorio, la tendencia actual es establecer zonas basadas en los umbrales tomando como valor importante el inicio o el valor previo (UL) a la acumulación del lactato sanguíneo y su relación con OBLA y MSLL.

De hallazgo importante para el eto es concluir que de entre las habituales tres vías duras (UAN-VO ${ }_{2 \text { máx }}-\mathrm{RAN}$ ) la que menos se entrena es la del UAN. Esta se entrena poco, pero se hace de forma concentrada en las semanas previas al evento principal. 
Diversos estudios confirman que, entre los fondistas de élite mundial, los grupos que realizaron ETO basado en cargas polarizadas (frente a los que predominan el volumen, el UAN o el HIT) obtuvieron, en general, mayores mejoras y adaptaciones de rendimiento, a largo plazo. Los estudios iniciados hace una década entre las universidades noruegas y el Comité Olímpico Noruego con los trabajos de Seiler y Kjerland, (2006) y Seiler y Tønnessen (2009) marcaron una nueva pauta de cómo hay que distribuir las cargas en deportes de resistencia. Desde entonces se establece que para llegar a los podios mundiales hay que cumplir la REGLA del 80-20, incluso aumentarla, ya que varios campeones o campeonas mundiales u olímpicos superan ese $80 \%$ llegando al $90 \%$ o más. El eto en zona UAN es muy escaso, generalmente no más del $5 \%$ del trabajo anual y justo antes de las grandes competiciones.

\section{Referencias}

Acevedo E., O. y Goldfarb A., H. (1989). Increased training intensity effects on plasma lactate,

ventilatory threshold, and endurance. Med Sci Sports Exerc,21, 563-568, Allen, H. y Coggan, A. (2010). Training and racing with a power meter. Boulder, USA: VeloPress.

Billat, V. L., Demarle, A., Slawinski, J., Paiva, M., y Koralsztein, J. P. (2001). Physical and training

characteristics of top-class marathon runners. Med Sci Sports Exerc. 33, 2089-2097.

Billat, V.L., Morton, R.H., Blondel, N., Berthoin, S., Bocquet, V., Koralsztein, J.P., y Barstow, T.J.

(2000). Oxygen kinetics and modelling of time to exhaustion whilst running at various velocities at maximal oxygen uptake. Eur. J. Appl. Physiol. 82:178-187.

Boudet, G., Garet, M., Bedu, M., Albuisson, E., y Chamoux, A. (2002). Median maximal heart rate for

heart rate calibration in different conditions: laboratory, field and competition. Int J Sports Med.

23(4), 290-297.

Bouzas Marins, J.C., Ottoline Marinsa, N.M., y Delgado Fernández, M. (2010) Aplicaciones de la

frecuencia cardiaca máxima en la evaluación y prescripción de ejercicio. Apunts Med Esport,45 (168):251-258. doi:10.1016/j. apunts.2010.04.003

Calderón, F.J., Benito, P.J., Peinado, A.B., y Díaz, V. (2008). Significado fisiológico de la transición

aeróbica-anaeróbica. Rev int med cienc act fís deporte, 8(32), 321-337.

Carazo-Vargas, P., y Moncada-Jiménez, J. (2015). A meta-analysis on the effects of exercise

training on the VO2max in children and adolescents. Retos. Nuevas tendencias en Educación Física, Deporte y Recreación, 27, 184-187

Carbonell Martínez, J.A., Ferrándiz Moreno, J., y Pascual Verdú, N. (2017). Análisis de la frecuencia cardíaca en el pádel femenino amateur. Retos. Nuevas tendencias en Educación Física, Deporte y Recreación,32, 204-207.

Chamari, K., y Padulo, J. (2015). «Aerobic» and «Anaerobic» terms used in exercise physiology: a

critical terminology reflection. Sport Medicine-Open, 1,9.

Chwalbinska-Moneta, J., Kaciuba-Uscilko, H., Krysztofiak, H., Ziemba, A., Krzeminski, K., Kruk, B., y

Nazar, K. (1998). Relationship between EMG blood lactate, and plasma catecholamine tresholds during graded exercise in men. Physiol Phyrmacol. 49, 433-441.

Coyle, E.F., Coggan, A.R., Hopper, M.K., y Walters, T, J. (1985) Determinants of endurance in well-

trained cyclists. J Appl Physiol;64: 2622-2630.

Coutinho, C., Watson, A., Brickson, S. y Sanfilippo, J. (2017). Maximal heart rate differs between

laboratory and field conditions among female athletes. Journal of Human Sport and Exercise, 12(2), 386-395. doi:10.14198/jhse.2017.122.15

Daussin, F.N., Ponsot, E., Dufour, S.P., Lonsdorfer-Wolf, E., Doutreleau, S., Geny, B.,... Richard, R.

(2007). Improvement of VO2max by cardiac output and oxygen extraction adaptation during intermittent versus continuous endurance training. European Journal of Applied Physiology. 101, 377-383 doi.org/10.1007/s00421-007-0499-3

Del Río Valdivia, J.E., Salazar, C.M., Romo, J.C., Andrade Sánchez, A.I., Flores Moreno, P.J., Barajas

Pineda, L.T., y Ramos Carranza, I.G. (2017). Diferencias en el OBLA en jugadoras de fútbol en relación a su posición en el campo de juego. Retos. Nuevas tendencias en Educación Física, Deporte y Recreación,32, 58-61

De Lucas, R.D., Dittrich, N., Junior, R.B., Kristopher M. De Souza, K.M., y Guglielmo, L.G.A. (2012) .

Is the Critical Running Speed Related to the Intermittent Maximal Lactate Steady State? J Sports Sci Med. 11(1): 89-94.

Denis, C., Dormois, D., y Lacour, J. R. (1984). Endurance training, VO2 max, and OBLA: a longitudinal

study of two different age groups. Int J SportsMed. 5, 167-173.

Esteve-Lanao, J., Foster, C., Seiler, S., y Lucia, A. (2007). Impact of training intensity distribution on

performance in endurance athletes. Strength Cond Res. 21(3) 943-949.

Evertsen, E, Medbo, J. I., y Bonen, A. (2001). Effect of training intensity on muscle lactate transporters and lactate threshold of cross-country skiers. Acta Physiol Scand. 173, 195-205.

Faude, O., Kindermann, W., y Meyer, T. (2009). Lactate threshold concepts: how valid are they?

SportsMed.39, 469-490.

Feliu J, Ventura, J.L., Segura, R., Rodas, G., Riera, J., Estruch, A., (...) y Capdevilla. (1999) Differences

between lactate concentration of samples from ear lobe and the fingertip. J Physiol Biochem, 55 (4): 333-9

Foster, C., Green, M.A., Snyder, A.C., y Thompson, N.N. (1993). Physiological responses during

stimulated competition. Med Sci Sports Exerc. 25(7), 877- 882

Gaskill, S. E., Walker, A. J., Serfass, R. A., Bouchard, C., Gagnon, J., Rao, D. C.,... Leon, A.S.(2001).

Changes in ventilatory threshold with exercise training in a sedentary population: the heritage family study. Int J Sports Med. 22, 586-592.

González Fimbres, R.A., Griego Amaya, H., Cuevas Castro, C.S., y Hernández Cruz, G. (2016).

Influencia del Volumen e Intensidad de la Carga de Entrenamiento en la Frecuencia

Cardiaca de recuperación. Retos. Nuevas tendencias en Educación Física, Deporte y Recreación,30,180-183

Guellich, A..y Seiler, S. (2010). Lactate profile changes in relation to training characteristics in junior elite cyclists. Int J Sports Physiol Perform. 5, 316-327.

Guro, S., Tønnessen, E., y Sandbakk, Ø. (2017). The Training Characteristics of the World's Most

Successful Female Cross-Country Skier. Frontiers in Physiology, 8, doi: 10.3389/fphys.2017.01069

Hall, M.M., S. Rajasekaran, S., Thomsen, T.W., y Peterson (2016). Lactate: Friend or foe. $P M R$; 8, S8-

S15. doi.org/10.1016/j.pmrj.2015.10.018

Hogan, M.C. (2001). Fall in intracellular PO2 at the onset of contractions in Xenopus single skeletal

muscle fibers. J. Appl. Physiol.90: 1871-1876.

Høydal, K.L. y Nord, I. (2017). The importance of heart rate monitors in controlling intensity during

training and competition in junior biathlon athletes. Journal of Human Sport and Exercise, 12(2), 358-366. doi:10.14198/jhse.2017.122.12

Ingham, S. A., Fudge, B. W., and Pringle, J. S. (2012). Training distribution, physiological profile, and

performance for a male international 1500-m runner. Int. J. Sports Physiol. Perform, 7, 193-195. doi: 10.1123/ijspp.7.2.193

Issurin. V. B. (2010). New Horizons for the Methodology and Physiology of Training Periodization.

Sports Med, 40(3): 189-206

Jeukendrup, A., y Diemen, A.V. (1998). Heart rate monitoring during training and competition in

cyclists. J Sports Sci.16(suppl 1), 91-99.

Kindermann,W., Simon, G., y Keul, J.(1979). The significance of the aerobic-anaerobic transition for the 
determination of work load intensities during endurance training. Eur $J$ Appl Physiol, 42, 25-34

Kunduracioglu, B., Guner, R., Ulkar, B., y Erdogan, A. (2007). Can heart rate values obtained from the

laboratory and field lactate tests be used interchangeably to prescribe exercise intensity for soccer players? Advances in Therapy, 24(4), 890-902.

Lajoie, C., Laurencelle, L., y Trudeau, F. (2000). Physiological responses to cycling for 60 minutes at

maximal lactate steady state. Can. J. Appl. Physiol,25, 250-261.

Lambert, M.I., Mbambo, ZH., y Gibson, A.S.C. (1998). Heart rate during training and competition for

long distance running. J Sports Sci,16 (Supp 1), 85-90.

Londeree, B. R. (1997). Effect of training on lactate/ventilatorythresholds: a meta-analysis. Med Sci

Sports Exerc, 29, 837-843.

Lopes-Motoyama, Y., Assis-Pereira, P.E., Jesús-Esteves, G., PereiraDuarte, J.M., Puibelli-Carrara,V.C.,

Mello-Risato, G., y Siva-Marques, P.H. (2013). Alternative methods for stimating maximum lactate steady state velocity in physically active young adults. Revista brasileira de cineantropometria e desmpenho humano, 16(4),419-426.

López Chicharro y Vicente Campos (2017). Umbral Láctico. Bases fisiológicas y aplicación al

entrenamiento. Madrid; España. Panamericana

Manunzio, C., Mester, J., Kaiser, W., and Wahl, P. (2016). Training intensity distribution and changes in

performance and physiology of a 2nd place finisher team of the race across America over a 6-month preparation period. Front. Physiol. 7:642. doi: 10.3389/fphys.2016.00642

Murillo Lorente, V., Álvarez Medina, J., y Manomelles Marqueta, P. (2016). Control de las cargas de eto a través de la percepción subjetiva. Predicción de la frecuencia cardiaca. Retos. Nuevas

tendencias en Educación Física, Deporte y Recreación,30, 82-86.

Navarro, F., Arellano, R., Carnero, C., y Gosálvez, M. (1990). Natación. Madrid, España:

Comité Olímpico Español.

Neal, C. M., Hunter, A. M., Brennan, L., O’ Sullivan, A., Hamilton, D.L., De Vito, G. et al.

(2013). Six weeks of a polarized training-intensity distribution leads to greater physiological and performance adaptations than a threshold model in trained cyclists. Appl Physiol.114, 461-471

Olbrecht, J., Madsen, O., Mader, A., Liesen, H., y Hollmann, W. (1985). Relationship between swimming

velocity and lactic concentration during continuous and intermittent training exercises. Int J Sports Med, 6(2): 74-7.

Platonov, V. (2001). Teoría General del Eto Deportivo Olímpico. Barcelona, España. Paidotribo.

Potteiger, J.A., y Weber, S.F. (1994). Ratings of perceived exertion and heart rate as indicators of

exercise intensity in different environmental temperatures. Med. Sci. Sports Exerc,26(6), 791-796.

Robergs, R.A., Chwalbinska-Moneta, J., Mitchell, J.B., Pascoe, D. D., Houmard, J., y Costill,

D. L. (1990). Blood lactate threshold differences between arterialized and venous blood. Int J Sports Med,11 (6): 446-51.

Rodríguez-Marroyo, J., Villa, J.G., García-López, J., y Foster, C. (2013). Relationship between the talk

test and ventilatory thersholds in well trained cyclist. Journal of strength and conditioning research, 27(7), 1942-9.

Roecker, K., Schotte, O., Niess, A.M., Horstmann, T., Dickhuth, H.H. (1998). Predicting competition

performance in long-distance running by means of a treadmill test. Med Sci Sports Exerc, (10):1552-7.

Sandbakk, O., Holmberg, H. C., Leirdal, S., and Ettema, G. (2011). The physiology of world-class sprint skiers. Scand J Med Sci Sports. 21, e9-el6.

Santos-Concejero, J., Granados, C., Bidaurrazaga-Letona, I., Zabala-Lili, J., Irazusta, J., y María Gil, S.

(2013). Onset of blood lactate accumulation as a predictor of performance in top athletes. Retos. Nuevas tendencias en Educación Física, Deporte y Recreación,23, 67-69.

Schumacher, Y. O., y Mueller, P. (2002). The 4000-m team pursuit cycling world record: theoretical and practical aspects. Med Sci Sports Exerc.34, 1029-1036.
Seiler, K. S., and Kjerland, G. O. (2006). Quantifying training intensity distribution in elite endurance

athletes: is there evidence for an «optimal» distribution? Scand. J. Med. Sci. Sports,16, 49-56. doi: 10.1111/j.1600-0838.2004. 00418.x

Seiler, S. y Tønnessen, E. (2009). Intervals, Thresholds, and Long Slow Distance: The Role of Intensity

and Duration in Endurance Training. Sport science, 13,32-53.

Semin K., Stahlnecker, A., Heelan, K., Brown, G., Shaw, B. y Shaw, I. (2008). Discrepancy Between

Training, Competition and Laboratory Measures of Maximum Heart Rate in Ncaa Division 2 Distance Runners. J Sport Sci Med, 7, 455 - 460 .

Sergeyevich-Mishchenko, V. y Dmitriyevich-Monogarov, V. (2001). Fisiología del Deportista. $2^{\mathrm{a}}$

Edición. Barcelona, España: Paidotribo.

Serrano Pérez, J. (2015). Metodologías de valoración del umbral anaeróbico aplicado al atletismo de fondo. Revisión bibliográfica (Trabajo Fin de Grado). Facultad de ciencias de la actividad física

y el deporte. Universidad Miguel Hernández. Alicante. España.

Skinner, J. S., y Mclellan, T.H. (1980). The transition from aerobic to anaerobic metabolism. Res $Q$ Exerc Sport,51, 234-48.

Stegmann, H., y Kindermann, W. (1982) Comparison of prolonged exercise tests at the individual

anaerobic threshold and the fixed anaerobic threshold of 4 mmol.l(-1) lactate. Int J Sports Med, 3, 105-110.

Steinacker, J. M., Lormes, W., Lehmann, M., y Altenburg, D. (1998). Training of rowers before world

championships. Med Sci Sports Exerc. 30, 1158-1163.

Stöggl, T.y Sperlich, B. (2014). Polarized training has greater impact on key endurance variables than

threshold, high intensity, or high-volume training. Frontiers in Physiology, $5,33$.

Stöggl, T. L., and Sperlich, B. (2015). The training intensity distribution among well-trained and elite

endurance athletes. Front. Physiol. 6:295. doi: 10.3389/fphys.2015.00295

Svedahl, K., y Macintosh, B.R. (2003). Anaerobic threshold: the concept and methods of measurement.

Can J Appl Physiol, 28(2), 299-323

Talanian, J. L., Galloway, S.D., Heigenhauser, G.J., Bonen, A., Spriet, L.L. (2007). Two weeks of high-

intensity aerobic interval training increases the capacity for fat oxidation during exercise in women. J Appl Physiol 102:1439-1447.

Tjelta, L. I. (2013). A longitudinal case study of the training of the 2012 European $1500 \mathrm{~m}$ track

champion. Int. J. Appl. Sport Sci. 25, 11-18. doi: 10.24985/ijass. 2013.25.1.11

Tonnessen, E., Svendsen, I. S., Ronnestad, B. R., Hisdal, J., Haugen, T. A., y Seiler, S. (2015). The

annual training periodization of 8 world champions in orienteering. Int. $J$. Sports Physiol. Perform,10, 29-38. doi: 10.1123/ijspp.2014-0005

Tonnessen, E., Sylta, O., Haugen, T. A., Hem, E., Svendsen, I. S., y Seiler, S. (2014). The road to gold: training and peaking characteristics in the year prior to a gold medal endurance performance.

PLOS ONE 9: e101796. doi: 10.1371/journal.pone.0101796

Van Schuylenbergh, R., Vanden Eynde, B., Hespel, P. (2004) Correlations between lactate and

ventilatory thresholds and the maximal lactate steady state in elite cyclists. Int J Sports Med;25: 403-408.

Vergès, S., Flore, P., y Favre-Juvin A. (2003). Blood lactate concentration/ heart rate relationship:

laboratory running test vs field roller skiing test. Int J SportsMe ,24(6):44651.

Versohansky, Y. (2007). The training system in middle distance running. Journal of Sport Strength Training Methodology. 3. December.

Wahl, P., Zwingmann, L., Manunzio, C., Wolf, J., y Bloch, W . (2018). Int. J. Sports Med, 39 (7) 541-

548. doi: 10.1055/s-0044-102131

Wasserman, K.,y Mcllory, M.B. (1964). Detecting the threshold of anaerobic metabolism in cardiac patiens during exercice. Am M Cardiol, 14, 844-852.

Zuniga, J.M., Housh, T.J, Camic, C.L., Bergstrom, H.C., Schmidt, R.J., y Johnson GO. (2014) The effect

of different exercise protocols and regression-based algorithms on the assessment of the anaerobic threshold. J Strength Cond Res;28: 2507-2512. 